\title{
Automatic compensation of tool disturbances, caused by an unstable cutting process
}

\author{
Vladimir Gusev ${ }^{1, *}$ \\ ${ }^{1}$ Institute of Engineering and Automobile Transport, Vladimir State University named under \\ Alexander and Nikolay Stoletovs, Russia
}

\begin{abstract}
Modern machining processes are characterized by a separate functioning of the elements of technological system (TS). The cutting mode, that determines the machining efficiency, depends on the functional capability of a low-performance element, and the other higher-performance elements are not able to reduce the workload of the first element. The "strong" element of the TS can not help the "weak" one in reducing the received workload. The transfer of a part of the workload from the "weak" to the "strong" element allows to use the higher cutting mode. In such statement the increase of efficiency of machining processes in the scientific and technical literature was not observed. Is offered to connect a pliable cutting tool with a rigid large-sized workpiece by special contact knot, which allows to compensate the tool perturbations, caused by unstable process of cutting. This knot provides a dissipative and elastic connection between the tool and the workpiece, has been made and tested at holes machining. As a result the increase of efficiency of machining processes of holes was installed.
\end{abstract}

\section{Introduction}

In the industry the large-sized details have a wide application, the weight of such details is measured by tens and hundreds kg. The worn out working surfaces of these details repair by a welding of a wear-resistant material and the next machining [1, 2]. The welded surface layer has significant geometrical roughness and high hardness [3, 4], therefore during machining a vibration of the TS elements occurs, which leads to a reduction of a work time of the cutting tool in 3-4 times [5], a reduction of the machining productivity. In works [616] raise a stability of machining by change of dynamic parameters of cutting tool.

Use of the tool with variable dynamic characteristics allows to reduce probability of origination of the most dangerous regenerative self-excited vibrations.

The analysis of works [3, 4, 17] testifies, that machining of weld surfaces occurs in extreme conditions and of named above measures insufficiently for significant increase of efficiency of repair manufacture. A weld surface is processed on the minimum cutting regimes at a big wear of an expensive cutting tool, what increase the technological cost

\footnotetext{
* Corresponding author: prof_gusev@mail.ru
} 
price of details [18]. To eliminate noted shortcomings is offered a new direction of raise of functional properties of the TS [19, 20].

\section{Direction of increase in the functional properties of the technological system}

It was previously noted, that the elements of technological system differ from each other in their static and dynamic properties: one of the elements (for example, a large-sized workpiece) has a high stiffness, vibration resistance, large mass, inertial and damping ability, but the other element (the cutting tool) is characterized by its low values. In such conditions, during the machining it is advisable to use the high physic-mechanical characteristics of the workpiece for increase of the stiffness and vibration resistance of the cutting tool. The implementation of such approach will improve the functional properties not only of the "weak" element, but also of the entire technological system.

\section{Substantiation of scheme of the elastic and dissipative connection of the tool and the workpiece}

To improve the functional properties of the cutting tool by using the properties of the workpiece, it was proposed to create an elastic and dissipative connection between them. For the machining of a hole 1 (Fig. 1) of workpiece 2 by the cutter 3 , we create these connection by inserting a special contact knot $(C K)$ in the $T S$, characterized by the stiffness $C$ and the dissipative ability $k$. At the machining, the tool 3 make a cutting motion in the arrow direction $D r$ and the feed motion - in the arrow direction $D s$.

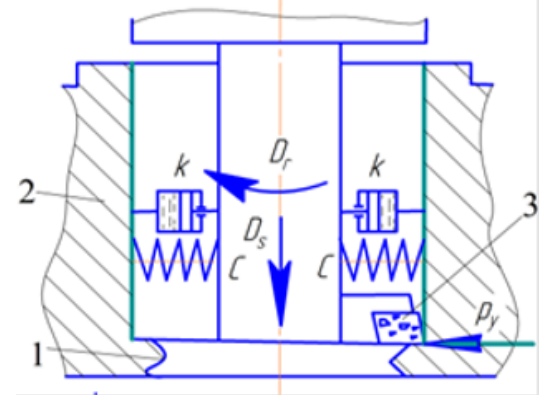

Fig. 1. Hole machining with an elastic and dissipative connection of the tool and workpiece.

The $C K$ is devoid of the cutting function and is designed to transfer external disturbances of the cutting tool to the workpiece. The stiffness $C$ of the contact knot is used for compensation of the elastic displacements of the cutting tool, and the damping properties (coefficient $k$ ) are used to reduce the oscillations, which depend on the first derivative of elastic displacements of the tool. Let's prove an offered way of decrease of vibration level of the TS by using of CK on the basis of the differential equation of the tool movement under the influence of a cutting force $P_{y}$ (Fig. 1). This equation has a view:

$$
M \frac{d^{2} y}{d t^{2}}+k \frac{d y}{d t}+C y=P_{y},
$$


where $M$ - is the oscillating mass; $\frac{d^{2} y}{d t^{2}}, \frac{d y}{d t}, y-$ are the acceleration, velocity and

displacement of the oscillating mass respectively; $k, C$ - are the damping coefficient and stiffness of the $C K$ respectively.

Because of communication of an tool with workpiece, the mass, participating in oscillatory process, can be accepted the equal:

$$
\mathrm{M}=m_{i}+m_{z},
$$

where $m_{i}, m_{z}-$ is the mass of the cutting tool and workpiece respectively.

The mass $m_{z}$ on one-two order is more, than the mass $m_{i}$, therefore the component $M \frac{d^{2} y}{d t^{2}}$ of the equation (1), that characterizes the inertial force, directed on a vibration decrease of the TS, much more, than at the traditional machining, at which CK is not used. Members $k \frac{d y}{d t}, C y$ also reduce the vibration level of the TS, and they are much smaller at traditional machining, because of the absence of the mentioned communications and the small values of $k$ and C.

Thus, at using in technological system the $C K$ all three members of equation (1) are significantly more in absolute magnitude, than the similar members for a technological system without $C K$, what provides a reduction of the TS oscillations, called by perturbations of the tool from the unstable cutting process. The cutting tool oscillations are reduced by a big mass of the workpiece (the inertial part of the oscillations reduction), the damping of contact knot (dissipative part), and stiffness of the $C K$ (elastic part).

\section{Practical realization of elastic and dissipative connections of the tool and workpiece}

The scheme of the elastic and dissipative connection of the tool and the workpiece was worked out (Fig. 2). In the hole of the spindle 1 the counterbore 2 is fixed.

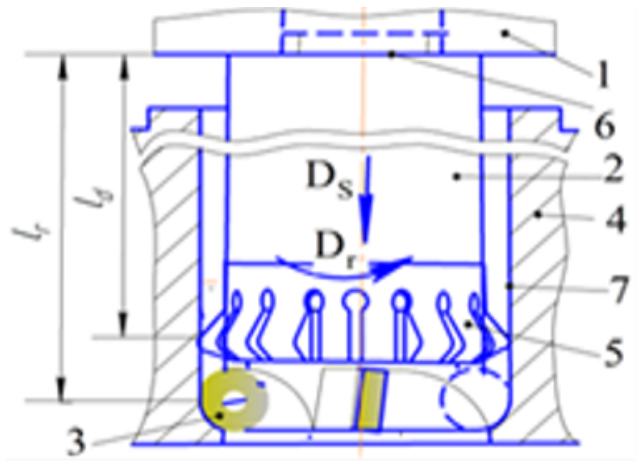

Fig. 2. The hole machining by a counterbore, equipped with a contact knot. 
The cutting plates 3 process the workpiece 4. Elastic damping element 5 (EDE) of the contact knot is located in close proximity to the cutting plates 3 . The distance $l_{d}$ from the end face 6 of the spindle 1 to the contact points of EDE with the hole 7 is less, than the distance $l_{r}$ of the contact points of the cutting plates 3 with the workpiece 4 . The contact knot consists of a bearing 1 (Fig. 3), whose inner ring 2 is pressed on the cylindrical surface 3 of the body 4 and fixed by the ring 5 .

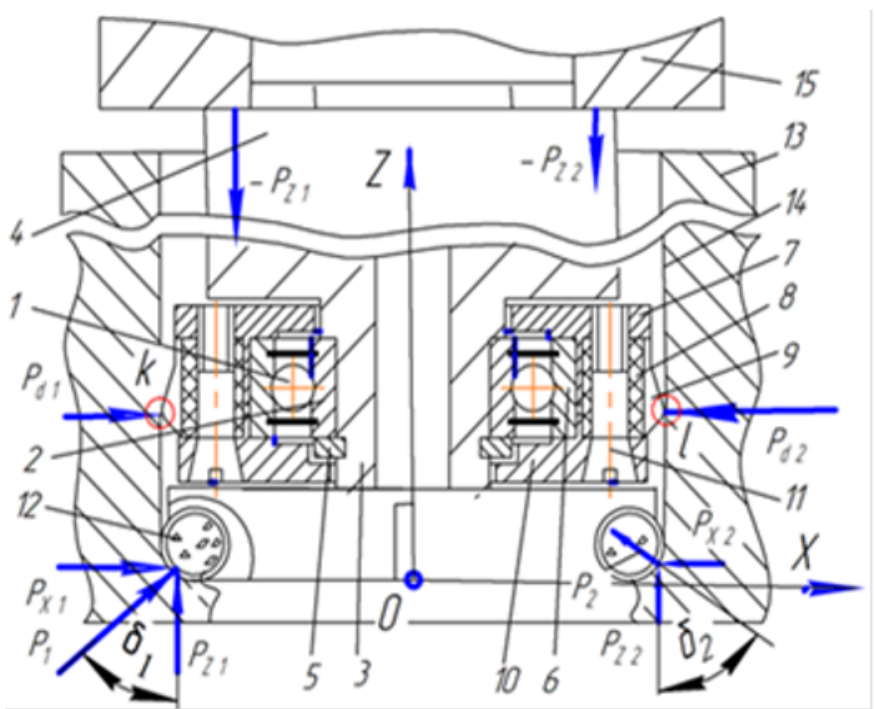

Fig. 3. Contact knot for automatic compensation of the tool disturbances [19, 20].

The elastic-damping element 7 (EDE) is installed on the outer ring 6 of the bearing 1 . EDE has an inner cavity, filled with damping material 8 (for example, with vulcanized rubber). That allows to compensate not only the tool displacement, but also the vibration velocity. The outer profile surface of the EDE 7 is devided on the elastic plates 9. EDE 7 is closed with the cover 10, which is tightened by screws 11 . The cutting plates 12 (Fig. 3) have a contact with the workpiece 13 and process the hole 14 . Owing to the changing of hole allowance, the cutting depth (for example, for the cutting plate 12), is larger compared to a diametrically placed cutting plate. In this case the cutting force $P_{1} \succ P_{2}$ and the angle $\delta_{1}$ of inclination of the force $P_{1}$ to the tool axis is different from the angle $\delta_{2}$ of inclination of the force $P_{2}$. Let's choose a system of coordinates XOZ, and project the cutting forces on the axis $\mathrm{X}$ and $\mathrm{Z}$, and we will get a result:

$$
\begin{aligned}
& \Sigma X=P_{x 1}-P_{x 2}=P_{1} \sin \delta_{1}-P_{2} \sin \delta_{2}, \\
& \Sigma Z=P_{Z 1}+P_{Z 2}=P_{1} \cos \delta_{1}+P_{2} \cos \delta_{2},
\end{aligned}
$$

where $P_{x 1}, P_{x 2}$ - is the horizontal component of the cutting force $P_{1}$ and $P_{2}$, respectively; $P_{z 1}, P_{z 2}$ - is the vertical component of the cutting force $P_{1}$ and $P_{2}$. 
The force $\Sigma X>0$ therefore, the more a difference of the cutting depth, the more a difference of forces and of a bending moment, acting on the counterbore. At the cutting process, a difference of forces $\left(P_{1} \sin \delta_{1}-P_{2} \sin \delta_{2}\right)>0$, which causes elastic displacements of the tool axis to the right. As a result the tightness in a point $k$ (Fig. 3) between the elastic plate 9 and the processed hole 14 decrease, and in the point $l$ - increase.

Under the action of the cutting force, the value of the elastic displacements of the tool axis in the cross section, which pass through the point of contact of the cutting plate 12 and a workpiece is equal to:

$$
X_{r}=\frac{\left(P_{1} \sin \delta_{1}-P_{2} \sin \delta_{2}\right) \cdot l_{r}^{3}}{3 E I}
$$

where $l_{r}$ - is the distance of the contact point of the cutting plate 3 (Fig. 2) with the hole 7 from the end face 6 of the spindle 1; $E$ - is the modulus of elasticity of the counterbore material; $I=\frac{\pi D_{p r}^{4}}{64}-$ is inertia moment of cross-section of the counterbore; $D_{p r}-$ is the resulted diameter of the counterbore.

The value of the elastic displacements of the counterbore axis in the cross-section, which pass through the points $k$ and $l$, is:

$$
X_{d}=\frac{\left(P_{1} \sin \delta_{1}-P_{2} \sin \delta_{2}\right) \cdot l_{r}^{2} \cdot l_{d}}{3 E I},
$$

where $l_{d}$ - is the distance of the point $k$ and $l$ from the end face 6 of the spindle 1 (Fig. 2).

The elastic displacements $X_{d}$ of the counterbore axis to the right lead to the changing in the displacements of the plate 9: in the point $k$ they decrease by $X_{d}$, and at the point $l$ they increase by $X_{d}$. The initial elastic displacements of the plates 9 after their entry into the hole 14 (when the cutting process is absente) are:

$$
X_{u}=0.5\left(D_{d}-D_{o}\right),
$$

where $D_{d}$ - is the maximum diameter of the EDE, when it is not inserted into the machined hole with a diameter $D_{O}$.

As a result of the counterbore axis bending, the elastic displacements of the plates 9 in the points $k$ and $l$ become equal to:

$$
\begin{aligned}
& X_{f k}=\frac{3 E I \cdot\left(D_{d}-D_{o}\right)-a_{1}}{6 E I}, \\
& X_{f l}=\frac{3 E I \cdot\left(D_{d}-D_{o}\right)+a_{1}}{6 E I} . \\
& a_{1}=2\left(P_{1} \sin \delta_{1}-P_{2} \sin \delta_{2}\right) \cdot l_{r}^{2} \cdot l_{d} .
\end{aligned}
$$


The workpiece through the elastic plates 9 acts on the cutting tool in the points $k$ and $l$ with the force:

$$
\begin{aligned}
& P_{k}=X_{f k} \cdot J_{p}, \\
& P_{l}=X_{f l} \cdot J_{p},
\end{aligned}
$$

where $J_{p}$ - is the plate 9 radial stiffness of the EDE 7 (Fig. 3).

The force, that was generated by the $C K$ and compensates the increment in the cutting force (external disturbance), is determined by the formula:

$$
\Delta P_{d}=\frac{2\left(P_{1} \sin \delta_{1}-P_{2} \sin \delta_{2}\right) \cdot l_{r}^{2} \cdot l_{d}}{3 E I} J_{p} .
$$

The full compensation of the force $\Sigma X$, that acts on the cutting tool, is provided when the equality is accomplished:

$$
\begin{gathered}
\Delta P_{d}=\Sigma X \\
\text { or } \quad \frac{2 \cdot l_{r}^{2} \cdot l_{d}}{3 E I} J_{p}=1 .
\end{gathered}
$$

From (14) we find the radial stiffness value, which the elastically plate 9 of the EDE 7 should have (Fig. 3) to compensate the force $\Sigma X$ :

$$
J_{p}=1,5 \frac{E I}{l_{r}^{2} \cdot l_{d}}
$$

By use of the formulas (3)-(15) solve the flat task of automatic compensation of external disturbance of the cutting tool. To solve the spatial task, it is necessary to consider the displacements of all elastic plates 9 , which contact with the processed hole 14 on the all circumference.

\section{Automatic compensation of the external disturbances of the cutting tool}

It was previously mentioned, that the elastic displacements of the cutting tool axis to the right side causes an increment in the displacements of the elastic plates 9 in the right half of the contact knot and a reduction - in the left half, what leads to a change of the elasticdamping forces, acting in contact of the plates with workpiece. The workpiece increases its action in the right half of the counterbore and reduces it in the left half, thus the force $P_{d 2}$ becomes more, then $P_{d 1}$ (Fig. 3). A momentary and automatic change of the elasticdamping forces in the $C K$ stabilizes the spatial position of the counterbore in relation to the hole at infringement of equality of the elementary cutting forces. On the counterbore also acts a force $\Sigma Z=\left(P_{1} \cos \delta_{1}+P_{2} \cos \delta_{2}\right)$, which is created by end face 6 of spindle. Spindle 
reaction occur in the form of forces $-P_{z 1},-P_{z 2}$ (Fig. 3), which compensate the forces $P_{1} \cos \delta_{1}, P_{2} \cos \delta_{2}$. Since the axial stiffness of the spindle is much more, than the radial stiffness of the counterbore, and the forces $P_{z 1}$ и $P_{z 2}$ have a comparatively small arm, equal to the radius of the processed hole, then the displacement of the counterbore axis under the action of the forces $P_{z 1}$ и $P_{z 2}$ can be ignored.

Thus, in case of equality infringement of the elementary cutting forces (at the appearance of an external disturbance) automaticaly arise in the contact knot the oppositely directed elastic and damping forces, which compensate the dynamic perturbations, generated by the unstable cut-friction process.

\section{Test of automatic compensation of an external disturbances of the cutting tool}

The counterbore, which realizes the interaction of the cutting plates and the $C K$ with the workpiece, is manufactured and tested in production conditions. On the FB-01 milling machine a conical hole of a large-sized beam of the railway car was processed. A counterbore without $C K$ (first variant) and a counterbore, equipped by $C K$ (second variant), were used. Both counterbores were equipped with cutting plates of the same dimensions and material. The tests showed, that in the first variant the counterbore provided only a partial removal of the allowance, which was welded to the conical hole. At the increase of the contact length of the cutting plates with the conical hole the vibration level increased rapidly, and the technological system entered into resonance, therefore a machining was stopping before the complete removal of the allowance, because of the misgivings of a breaking of the cutting plates. The machining of a conical hole in the second variant went quietly with a relatively low increment in the vibration level of the technological system. The tool removed all allowance of conical hole, providing the required quality of the machined surface.

Thus, the use of a cutting tool, equipped with an automatic system of compensation of external disturbances, increments the machining efficiency of the internal cylindrical surfaces and opens new researches directions in increase of productivity and quality of a flat and external cylindrical surfaces.

\section{Conclusion}

1. Elements of modern technological systems for machining differentially accept a working load, what leads to a reduction of the processes efficiency because of a need to set the minimum cutting mode, determined by the weakest element of the system

2. It is proposed to increase the machining efficiency by transferring the workload from an element with low to an element with high performance characteristics, what is achieved by the introducing into the technological system of elastic and dissipative connection between the cutting tool and the workpiece. This allows to significantly increase the cutting mode without of resonance occurrence in technological system

3. A main scheme has been developed and a real model of the cutting tool, equipped with a contact knot, was made, which automatically compensates the external disturbances of the tool from the unstable cutting process.

4. The mechanism of automatic compensation of external disturbances of the cutting tool is uncovered and the production tests of the contact knot has been carried out. The 
production tests confirmed the high efficiency of machining with using of the new cutting tool.

\section{References}

1. R. O. Koshkalda, etc. The design and repair of freight cars. Publishing house UMK of Ministry of Russia Railways, (2000). Internet resource: .https://studwood.ru/1681933/tehnika/neispravnosti_remont_nadressornyh_balok.

2. Processing of weld surfaces. Internet resource: https://studopedia.su/10_106369_obrabotka-naplavlennih-poverhnostey.html.

3. V. G. Gusev, Effective technology of processing surfaces of case details, RU, J. High technologies in mechanical engineering, No 2, (2018)

4. V. G. Gusev, Machining of weld surfaces with the cutting tool, equipped with the stabilising module, RU, J. High technologies in mechanical engineering, No 9 (87), (2018)

5. S. A. Vasin, The forecast of vibration stability of the tool at turning and milling, Moscow, J. Mechanical engineering, (2006)

6. L. A. Vasin, S. A. Vasin, and A. A. Koshelev, The emerdzhentnyj approach to creation of steady against vibration cutting tools, RU, J. News of the Tula State un-ty, (2014)

7. Patent 70471, RU, (2008)

8. Patent 72427 RU, (2008)

9. Patent 2457077, RU, (2012)

10. Patent 2479385, RU, (2013)

11. Patent 2535196, RU, (2014)

12. E. J. Gorin, A. J. Krjazhev, E. J. Tatrkin., etc. Processing improvement of quality at milling by use shakeproof designs of a face mill, Internet resource: http://elib.altstu.ru/journals/Files/pv2015_02/pdf/043gorin.pdf.

13. E. S. Ognevenko, Theoretical bases of decrease in vibrations level at use of edge tools with damping elements, Materials of XLVII MSPK, No 6(43), RU, Novosibirsk, (2015)

14. I. A. Sharapin, Calculation of drive fluctuations. Vibration insulation: studies the grant for students of technical colleges, RU, St.-Petersburg, (2014).

15. N. V. Novikov, etc., Influence of a cutting plates damping from polycrystals KH5 on a cutters durability, Ukr, Kiev, No 58, (2010)

16. V. V. Malyhin, E .I. Jatsun, S. G. Novikov, "Increase of operational characteristics of damping cutters, RU, Kursk, No 2, (2012).

17. V. G, Gusev, A. M. Selivanov, The cutting tool for regenerative processing of workpieces with a non-uniform allowance, Mat. of the Int. sci.-prac. conf., RU, Kursk, (2011)

18. V. G, Gusev, A.V. Lazarev, A. M. Selivanov, Modelling of elastic movings of cutting plates at core drilling apertures in program Cosmos Works, RU, J. Fundamental and applied problems of technics and technology, No 2-6 (292), (2012)

19. Patent 2457926, RU, (2012)

20. Patent 2615965, RU, (2017) 